\title{
Mining the Web for Visual Concepts
}

\author{
Nick Morsillo, Chris Pal, Randal Nelson \\ Department of Computer Science, University of Rochester \\ \{morsillo, pal, nelson\}@cs.rochester.edu
}

Univ. of Rochester Comp. Sci. Dept. Technical Report TR-2008-931

March 18, 2008

\begin{abstract}
The web has the potential to serve as an excellent source of example imagery for visual concepts. Image search engines based on text keywords can fetch thousands of images for a given query; however, their results tend to be visually noisy. We present a technique that allows a user to refine noisy search results and characterize a more precise visual object class. With a small amount of user intervention we are able to re-rank search engine results to obtain many more examples of the desired concept. Our approach is based on semi-supervised machine learning in a novel probabilistic graphical model composed of both generative and discriminative elements. Learning is achieved via a hybrid expectation maximization / expected gradient procedure initialized with a small example set defined by the user. We demonstrate our approach on images of musical instruments collected from Google image search. The rankings given by our model show significant improvement with respect to the user-refined query. The results are suitable for improving user experience in image search applications and for collecting large labeled datasets for computer vision research.
\end{abstract}

\section{Introduction}

With the advent of the internet we are accustomed to having practically any information we want within moments of a search query. Search engines have become strikingly accurate at delivering relevant text-based information from the web. The same cannot be said for web image content. There are billions of images on the web but most of them are not easily retrievable.

Current web image search tools leave plenty of room for improvement. Their accuracy rates typically range from about twenty to forty percent depending on the query. For example, Figure 1 displays the top 20 images returned by Google image search for the query "saxophone". These results exhibit some common symptoms of web image search technology: the image labels are very noisy and the relevance of each result is highly variable. The results are sometimes adequate but often far from desirable. We would like to have the same level of performance for visual content search on the web as we do for text content.

One way to improve web image search is one to look within the images for visual concepts and features of interest. This approach can be further enhanced by allowing a "visual query" to be specified by a search user. There are many ways that the visual query can be provided; 

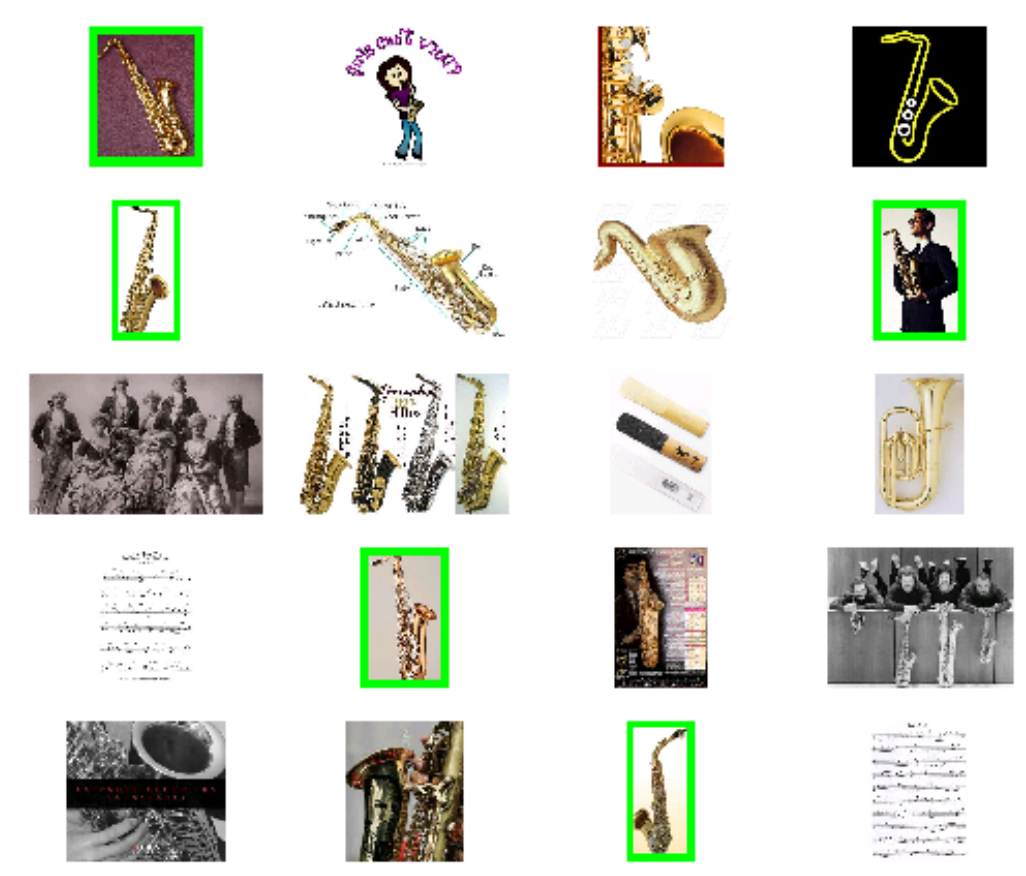

Figure 1: Top 20 results of a Google image search query for "saxophone" with user specified refinements indicated with green outlines.

for instance, a user could provide a sketch, upload an example image, or try to define the visual concept with words. We propose that there is a much easier, more natural way for the visual query to be specified. One can use existing image search technology to provide the user with a rough set of images from which he or she selects a small number of images depicting the visual concept of interest.

Once a user query is captured in the form of one or a few example images, the task becomes a matter of filtering the noisy web image search data to discover visually related images. Since the example images chosen by the user were selected from the web, they are associated with html documents and we can use these documents to learn a textual representation of the visual query. We can therefore construct a model which combines text and image features to mine the web for better image search results.

The model we present is unique for the following reasons. It is a tailored probabilistic graphical model containing both directed and undirected components in order to handle different feature types effectively. We explicitly handle the difficult task of learning from minimal training data (the small set of user query images) by developing a semi-supervised machine learning technique based on a novel, hybrid expectation maximization / expected gradient [7, 4] procedure for the model. Our approach has the added benefit that it is fast and fits nicely within the framework of existing image search technologies.

We show in our experiments that images returned by our model are significantly more accurate than traditional image search with respect to the user query. We find that both text and image 
features are useful, sometimes to different degrees depending on the query. Additionally, we show that our semi-supervised approach yields significant performance gains in this setting.

\section{Related Work}

The prospect of learning visual concepts from web data has started to receive considerable research attention [15, 18]. For example, Ponce et al. [15] emphasize the general need for better object category datasets. Our approach here as well as the others we briefly review represent parts of the solution to this problem. In the following review we emphasize recent work relevant to the problem of learning visual concepts from noisy search results. This problem can be viewed as synonymous with the goal of extracting a clean set of example images from noisy results for the purposes of dataset construction.

Fergus et al. [6] examine the problem of learning object categories from Google data. They develop a variant of the pLSA clustering method to successfully learn category models from noisy web data. An interesting component to this work is their method of selecting training data. They pick the top few image search results as the training set as the top results tend to be more correct. To collect even more potential training images they submit their query in multiple languages. Li et al. [9] also take an image-only approach. They use a hierarchical Dirichlet process [17] and demonstrate performance by retrieving clean image sets for a number of categories. [16] is another recent work which successfully retrieves clean image sets using image content alone.

Berg and Forsyth [2] look at a combination of text and image features focusing on animals for the dataset. They do a pre-clustering on the text via LDA, and their supervision is to pick the correct cluster. We find that this procedure did not work for many of our object classes because our experiments didn't exhibit an immediately clear text cluster and visual group correspondence.

At the same time as this increasing interest in leveraging the web to collect image datasets, there has been an increasing focus in the Machine Learning and Data Mining communities on the tradeoff between generative and discriminative approaches to pattern recognition $[13,11,8]$. A generative probabilistic model attempts to capture the joint probability distribution of data points $x$ and labels $y$, whereas a discriminative approach models only the conditional $p(y \mid x)$. Under the correct model assumptions and data conditions one approach can perform significantly better than the other. In general, generative models tend to succeed when the model makes fair independence assumptions about the data and the data is sparse. Discriminative approaches are better when the underlying data distribution $p(x)$ is difficult to model, or when the dataset size grows toward infinity. Our technique applies each of these approaches on portions of the data where they are expected to perform best using a probability model with both discriminative and generative components for different features. Our model is also principled in the sense that it makes no ad-hoc modifications to a likelihood objective defined by an underlying probabilistic model. 


\section{A Model for Web Images and Text}

\subsection{Image and Text Features}

For image features we use counts of quantized SIFT descriptors [10], commonly referred to as the bag-of-words representation. 2000 SIFT decriptors are sampled at random scales and locations for each image. We forego the the common approach of interest point detection in light of recent evidence that it is not necessarily more effective than random sampling [14]. Furthermore, we would like our system to handle a diversity of visual concepts, and keypoint detectors can have trouble with more uniform visual elements where stable interest points are difficult to locate. For a given image query we build a visual codebook of 400 words by application of k-means to a random sampling of 50,000 SIFT descriptors from multiple images. Each descriptor is assigned to its nearest word in the codebook, and an image is represented as a 400-vector of visual word counts.

Text features derived from associated html documents are given by the cluster assignments of the Latent Dirichlet Allocation document-topic model [3]. LDA is a generative model of documents where each document is a multinomial distribution over a set of latent topics, and each topic is a multinomial over words. It has been applied successfully to a number of modeling problems across many domains including computer vision [1]. Our application of LDA serves the purpose of dimensionality reduction.

Text processing begins by extracting the 100 words closest to the image anchor of interest. The words are filtered by a standard stoplist which is augmented to remove common html-related junk words. The words for each document are accumulated into an unordered collection of counts and put into vector form, a representation which is now identical to that of the visual features. We use the LDA implementation from the Mallet Toolkit [12] to train a model with 10 topics on the set of word vectors. Then, each document is represented as the 10 -vector of mixing proportions for the 10 topics.

Principal component analysis (PCA) is commonly applied to real valued data to both reduce the dimensionality of input data and to reduce correlations among the dimensions of the transformed data. As such, simpler models with feature independence assumptions (such as diagonal covariance Gaussian mixture models) can then be applied in the transformed space where independence assumptions are more valid. In a similar spirit, we use LDA to reduce the vocabulary size of a bag of words representation - with typically tens of thousands of words - to a small number of topics. Models developed for data expressed in a topic representation can thus more justifiably make independence assumptions.

In the model we describe in the next section, we use these image and text feature representations. While our preliminary experiments led us to select these particular feature representations, our probabilistic model is easily applied to any feature representation that has been transformed into a vocabulary or topic representation.

\subsection{The Model}

Our model consists of a principled combination of generative and discriminative elements. We model the features of $N_{i}$ images with corresponding web text. Let $h$ be a binary random variable indicating if a given image retrieved from a query should indeed be be associated with a user defined concept of interest, such that $h \in\{$ "relevant", "not relevant" $\}$. Let a represent a binary random 
variable for a match of the query string with a sub-string in the image filename. Let $b$ represent another binary random variable, indicating if there is an exact substring match in the html url.

We treat the raw words in a region surrounding the anchor text for the image as a set of random variables which we analyze using an LDA based topic model. From this topic representation we are thus naturally able to characterize each document as consisting of $N_{w}$ draws from a smaller vocabulary of topic words $W=\left\{w_{1}, w_{2}, \ldots, w_{N_{w}}\right\}$. SIFT features are quantized into a visual vocabulary as discussed in Section 3.1. Let the set of quantized SIFT features for a given image be represented as a set of visual words $V=\left\{v_{1}, v_{2}, \ldots, v_{N_{v}}\right\}$.

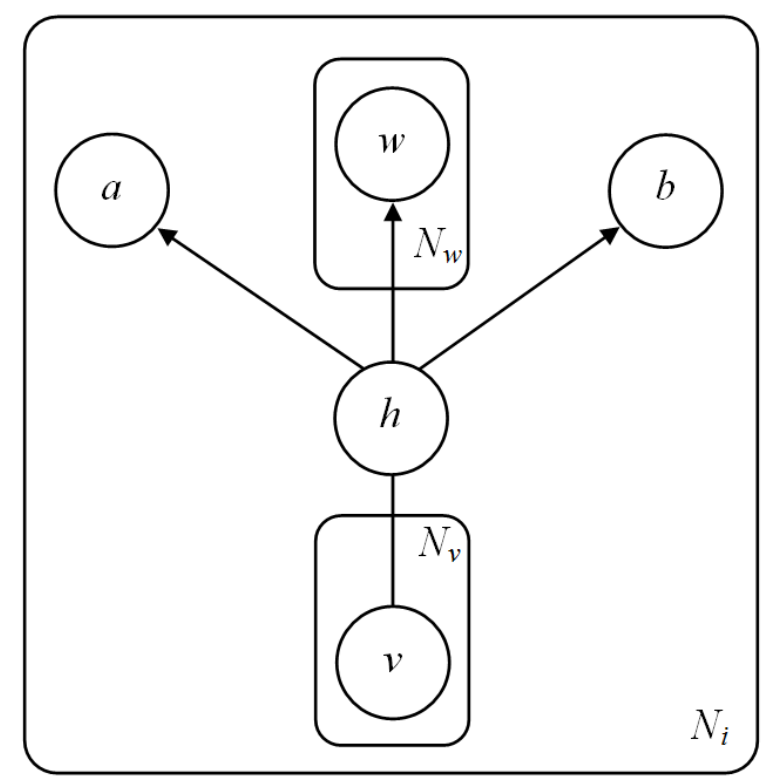

Figure 2: A graphical model with both generative (directed arrows) and discriminative elements (undirected arrows).

We shall use $X$ to denote the set of all random variables other than $h$ for which our model encodes a joint probability distribution, $X=\{a, b, W\}$. As we shall soon see, our model encodes a generative distribution on $X$ when conditioned on $V$. With the above definitions in place we use the model illustrated in figure 2 given by

$$
\begin{aligned}
P(X, h \mid V) & =P(a, b, W \mid h) P(h \mid V) \\
& =P(a \mid h) P(b \mid h) P(W \mid h) P(h \mid V) .
\end{aligned}
$$

For $P(h \mid V)$ we use a simple conditional random field

$$
P(h \mid V)=\frac{1}{Z(V)} \exp \left\{\sum_{k=1}^{K} \lambda_{k} f_{k}(h, V)\right\} .
$$

For (2) we define $k$ feature functions $f_{k}(h, X)$. In our experiments here, function $k$ evaluates to the number of counts for visual word $k$ for each of our $N_{v}$ possible visual words. For $P(a \mid h)$ and $P(b \mid h)$ 
we use binary distributions, and for our topical text words $P(W \mid h)$ we use a discrete distribution with the factorization

$$
P(W \mid h)=\prod_{i=1}^{N_{w}} P\left(w_{i} \mid h\right) .
$$

Let the complete parameter space be denoted as $\boldsymbol{\Theta}=\left\{\lambda, \theta_{a}, \theta_{w}, \theta_{b}\right\}$, corresponding to a partitioning of parameters for each of the respective components of the underlying graphical model.

\subsection{Estimation with Labeled Data}

When we have hand specified labels for $h$, our approach decomposes into separate estimation problems for the generative and discriminative components of the model. Our objective for labeled data is

$$
\log P(a, b, W, h \mid V)=\log P(a, b, W \mid h)+\log P(h \mid V) .
$$

The generative component of our model involves

$$
\begin{aligned}
\mathcal{L}_{X \mid h} & =\log P(a, b, W \mid h)=\sum_{i=1}^{N_{w}} \log P\left(w_{i} \mid h\right) \\
& +\log P(a \mid h)+\log P(b \mid h),
\end{aligned}
$$

affording closed form updates for the parameters of conditional distributions for $P(X \mid h)$ easily computed from the standard sufficient statistics for Bernoulli, Discrete and Multinomial distributions. In contrast, parameter estimates for discriminative or undirected components of our model are obtained iteratively by gradient descent using

$$
\begin{gathered}
\mathcal{L}_{h \mid V}=\log P(h \mid V)=\sum_{k=1}^{K} \lambda_{k} f_{k}(h, V)-\log Z(V) \\
=\lambda^{T} \mathbf{f}(h, V)-\log Z(V) \\
\frac{\partial \mathcal{L}_{h \mid V}}{\partial \lambda}=\mathbf{f}(h, V)-\sum_{h} \mathbf{f}(h, V) P(h \mid V)
\end{gathered}
$$

\subsection{Estimation with Unlabeled Data}

In this section we present a hybrid expectation maximization / expected gradient procedure to

perform learning with unlabeled data. For unlabeled data we wish to perform optimization based on the marginal probability

$$
p(a, b, W \mid V)=\sum_{h} P(a, b, W \mid h) P(h \mid V)
$$

Our objective is thus

$$
\mathcal{L}_{X \mid V}=\log \sum_{h} P(X \mid h) P(h \mid V)
$$


and parameter estimation involves

$$
\frac{\partial \mathcal{L}_{X \mid V}}{\partial \theta}=\sum_{h} P(h \mid X, V) \frac{\partial}{\partial \theta}[\log P(X \mid h)+\log P(h \mid V)] .
$$

We thus perform our optimization by computing an expectation or E-step followed by: (1) a single closed form maximization or M-step for parameter estimates involving variables in $X$

$$
\Theta_{v_{j} \mid h}=\frac{\sum_{i=1}^{|D|} P\left(h_{i} \mid V_{i}, X_{i}\right) N\left(v_{j, i}\right)}{\sum_{i=1}^{|D|} \sum_{s=1}^{|X|} P\left(h_{i} \mid V_{i}, X_{i}\right) N\left(v_{s, i}\right)}
$$

and (2) an iterative expected gradient descent optimization (until local convergence) for the discriminative component of the model

$$
\frac{\partial \mathcal{L}_{h \mid V}}{\partial \lambda}=\sum_{h} \mathbf{f}(h, V) P(h \mid X, V)-\sum_{h} \sum_{X} \mathbf{f}(h, V) P(h, X \mid V)
$$

The complete optimization procedure thus consists of iterations of: an E-step followed by an Mstep and an expected gradient optimization, repeating these steps until the likelihood change over iterations is within a relative log likelihood change tolerance of .001 .

When we have a mixture of very small number of positive example images among many unlabeled instances, semi-supervised techniques can be sensitive to the precise selection of labeled elements [5]. In our approach here whenever we have a mixture of labeled and unlabeled data we train our model on the labeled data first to obtain initial parameter estimates prior to a more complete optimization with both the labeled and unlabeled data. In our experiments here we also use a random sampling procedure to simulate user specified labels. However, in the context of a real world user interface to customized image search, the set of labeled examples likely has a better chance of being more representative of the variability in a users definition for any given visual concept.

\section{Experiments}

In the following section we describe experiments with our model on a web dataset themed by musical instruments. First we describe our acquisition and processing procedure for the dataset. We then evaluate model performance with respect to different visual queries and varying levels of supervision. In addition we show that the text+vision model with carefully initialized expectation maximization yields better performance than a series of baseline experiments.

\subsection{Dataset Acquisition}

We are unable to find a commonly used, publicly available dataset containing both web image and text data suitable for our experiments. Therefore we turn to Google image search to acquire our dataset.

Table 1 lists our choice of query words which define the visual categories of the dataset. We choose a theme of musical instruments for the following reasons. Musical instruments are typically 


\begin{tabular}{|c|c|c|}
\hline Query string & Instances & True instances \\
\hline french horn & 549 & 112 \\
\hline harp & 531 & 93 \\
\hline harpsichord & 394 & 113 \\
\hline piano & 503 & 59 \\
\hline saxophone & 521 & 102 \\
\hline timpani & 480 & 37 \\
\hline tuba & 487 & 67 \\
\hline violin & 527 & 130 \\
\hline xylophone & 495 & 58 \\
\hline \hline
\end{tabular}

Table 1: Musical instruments web dataset.

well defined as visual categories, and this makes performance evaluation on the dataset less subjective. Additionally, instruments represent the sort of web query that search engine operators and users would want to refine, particularly in an e-commerce setting. Our choice of a themed dataset rather than a disparate one (e.g. the categories of Caltech 101) facilitates future experiments of forced-choice classification between a challenging set of objects which tend to co-occur in the real world.

For each of the categories in table 1 we fetch the top 1000 results from Google image search using the category name as the query. For each result we capture the image and the associated html document, as well as the image and html urls and filenames, and the Google rank order. We filter the results to remove grayscale images, empty or damaged images, and instances with missing data. Table 1 lists the number of elements in each category after filtering.

We further process each instance by pre-computing the image and text features to be used by the model. Images are scaled to contain roughly the same number of pixels (about 320x240) while maintaining aspect ratio. As discussed previously, 2000 SIFT descriptors are computed at random scales and locations in each image. A visual vocabulary with 400 entries is built for each class via $\mathrm{k}$-means, and a vector of word counts is recorded in standard bag-of-words fashion. Text features are computed as a vector of latent topic mixing proportions as described in previous sections.

Our performance evaluations require us to know the ground truths for each image. In our scenario the truth labels represent the desired assignment of the variable $h$ in the model. A difficulty arises when we create human labelings of images from the web: we must define what the desired visual concept is for each class, and we must deal with images that have ambiguous meaning with respect to $h \in\{$ "relevant", "not relevant" $\}$ in a consistent way. We define the following set of rules to make our labelings consistent:

- The visual concept of interest is a clear, realistic image of the most common form of the object defined by the query word.

- The visual concept must be the focus of the image. We reject images where there are more than 3 prominently displayed objects including the object of interest. The object of interest must be at least as prominent in the image as any other object or theme. 


\begin{tabular}{|c|c|c|c|c|c|}
\hline Query string & Full Model & no-EM & vision-only & text-only & Google \\
\hline french horn & $\mathbf{0 . 9 2}$ & 0.80 & 0.82 & 0.56 & 0.70 \\
\hline harp & 0.62 & $\mathbf{0 . 7 0}$ & 0.40 & 0.58 & 0.60 \\
\hline harpsichord & $\mathbf{0 . 9 4}$ & 0.90 & 0.90 & 0.78 & 0.60 \\
\hline piano & $\mathbf{0 . 8 0}$ & $\mathbf{0 . 8 0}$ & 0.50 & 0.68 & 0.70 \\
\hline saxophone & $\mathbf{0 . 9 0}$ & 0.78 & 0.78 & 0.80 & 0.70 \\
\hline timpani & $\mathbf{0 . 8 6}$ & 0.70 & 0.90 & 0.56 & 0.60 \\
\hline tuba & $\mathbf{0 . 9 8}$ & 0.94 & 0.90 & 0.56 & 0.50 \\
\hline violin & $\mathbf{0 . 8 6}$ & 0.72 & $\mathbf{0 . 8 6}$ & 0.34 & 0.70 \\
\hline xylophone & 0.78 & $\mathbf{0 . 8 0}$ & 0.68 & 0.74 & 0.40 \\
\hline \hline
\end{tabular}

Table 2: Top-10 accuracy for each model and class. Top-10 accuracy represents the proportion of images correct among the top-10 ranked images by our model, averaged over multiple trials with different training sets. We note that in cases where accuracy is low, false positives are typically quite reasonable as demonstrated in Figure 5.

- We reject images containing more than 3 instances of the object of interest. With many repeated instances of an object, the visual concept changes and begins to resemble a texture.

- We reject cartoon images and abstract art. Photorealistic images are retained as long as they are convincing to a human observer.

- If the context surrounding the object seems bizarre or impossible to a human observer, the image is labled as background.

- Extreme close-ups, distant views, and majority occlusions are rejected. Most of the the object should be clearly visible.

- We include images of objects which are sufficiently similar as to fool a human observer (e.g., we include images of mellophones with the french horn class).

The dataset including all computed features and truth labelings is made publicly available on the web. We feel that a common dataset is needed for future research of image mining using both vision and text, in order to provide the research community with a means for common performance comparison.

\subsection{Performances for 9 queries}

Here we wish to demonstrate the efficacy of our model over a range of visual concepts. We use precision-recall as our measure and we compare to the ranking given by the original image search engine. We run a set of 10 repeated experiments for each of the classes listed in table one. For each repeat under a given class we vary the training set (corresponding to example images selected during user intervention) by randomly choosing 5 images from the pool of true-class images. We 

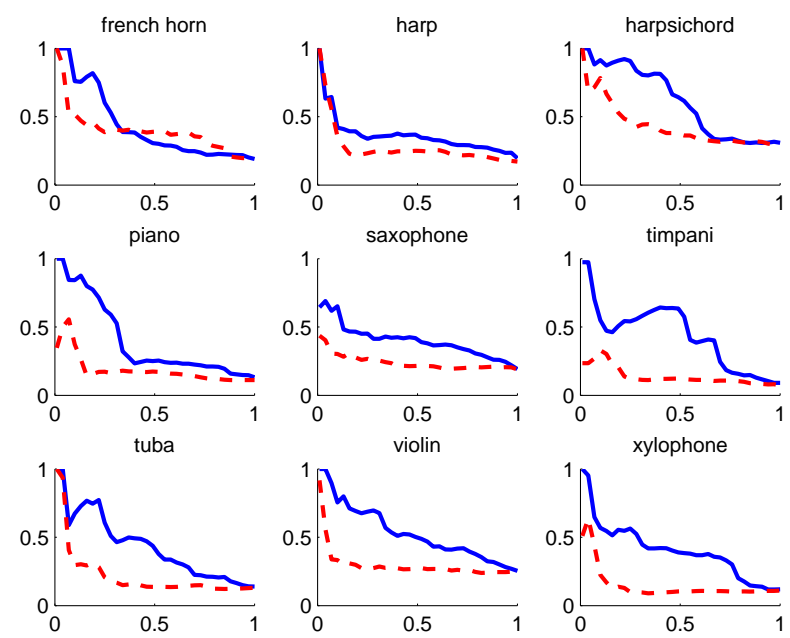

(a)

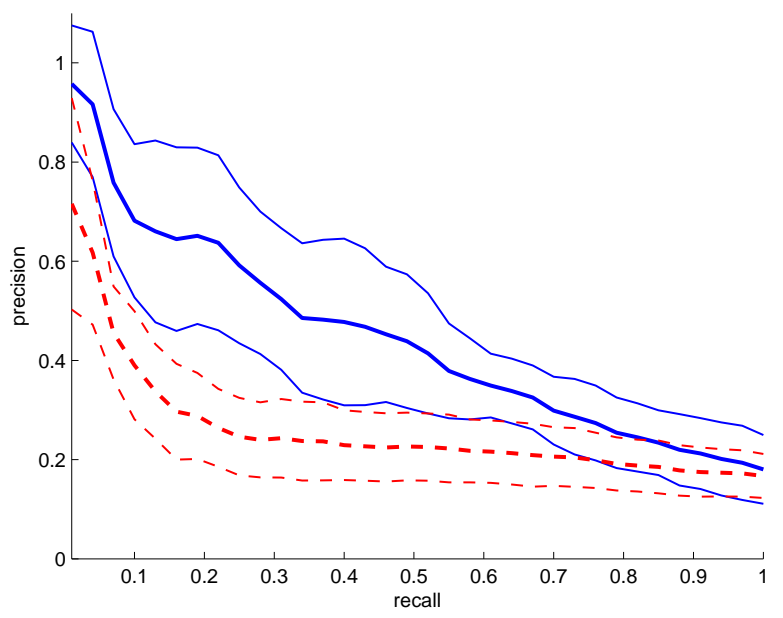

(b)

Figure 3: (a) gives the precision-recall curves for each of the categories in our web dataset. (b) provides the average precision-recall $\pm \sigma$ over the entire dataset. Solid blue curves represent the performances of our model, and dashed red curves give the performance of Google rankings.

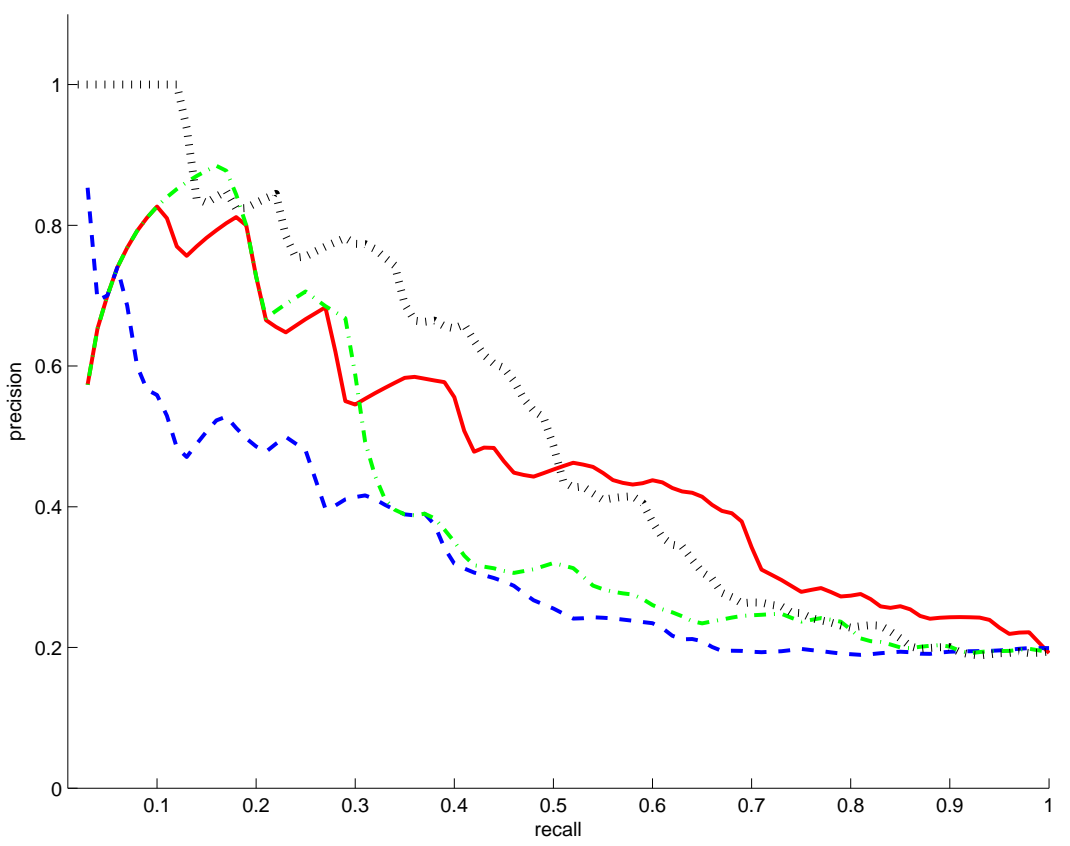

Figure 4: Performance comparison to various baseline models using the saxophone class. Black dotted line is our standard model with semi-supervised learning. Solid red line is our model without semi-supervised learning. Green dash-dotted line is image-only model, and blue dashed line is text-only model. 


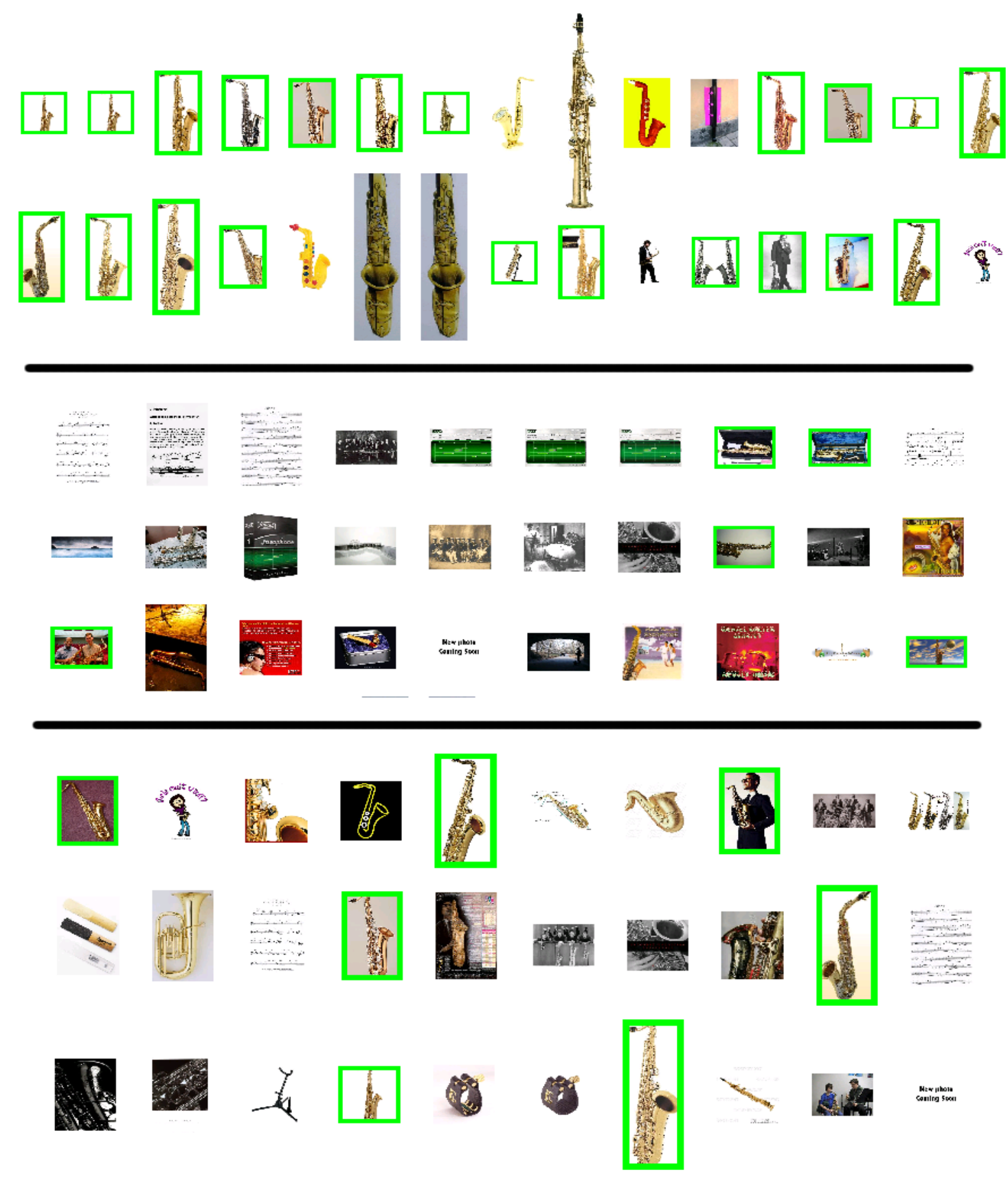

Figure 5: Sample results of our model for the saxophone class. Green borders indicate dataset entries which were pre-labeled as true. The first two rows show the top 30 results as ranked by our model. Images in the middle 3 rows are the images ranked lowest by our model, and the last 3 rows give the top 30 search engine results for comparison. We see that the proposed model returns much cleaner visual concepts and appropriately discards unrelated images. The occasional false positives given by the model are still visually relevant to the query. 
then run the hybrid semi-supervised algorithm until convergence, which typically occurs within 30 iterations.

Figure 3 shows the average precision-recall curve for each class given by the model and by the Google rank. We note that our model outperforms the original search engine ranking for every class, sometimes significantly so. Search engine results are typically more accurate for the highest-ranked elements, and we see the same effect in our model rerankings. The top set of images returned is generally very clean and would be suitable as datasets for computer vision experiments. Figure 5 displays the top 30 results returned by our model for the saxophone class, and compares these results to the top 30 returned by Google.

\subsection{Comparisons to baseline methods}

It was shown in [2] that for some classes, text features are more reliable predictors for classification, while for other classes visual features are more useful. This makes intuitive sense and we wish to validate this argument for our model. We compare the performances of a number of variants of our model to judge the merits of its various components.

The model variants we tested include the model without the hybrid expectation maximization procedure, a portion of the model using only text features, and a portion of the model using only image features. In these tests we again use 5 randomly chosen positive training examples.

Indeed, we find that for some classes text is more important while for others visual features are better. The combination of features in our model yields better performance in almost every case. Additionally, performing the hybrid expectation-maximization procedure further improves results. Figure 4 gives the performance comparison of the model variants for the saxophone class. Table 2 lists the accuracy among the top-10 ranked images for each method and each class.

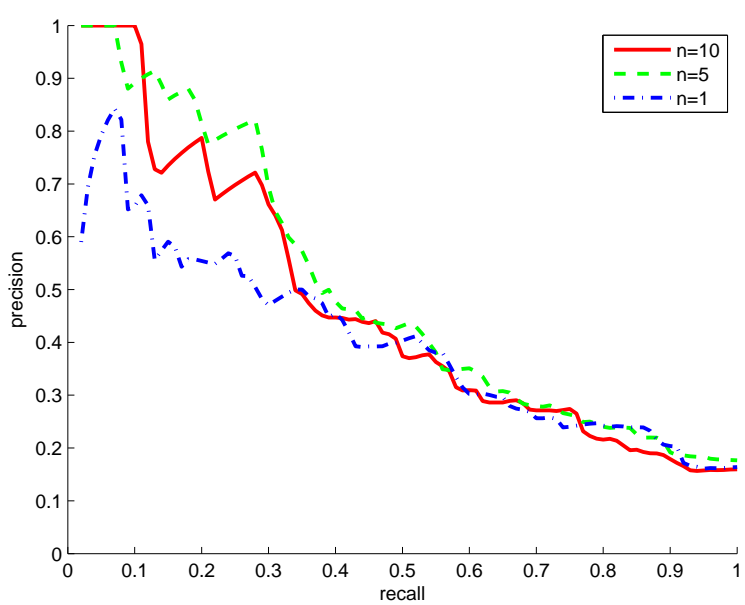

(a)

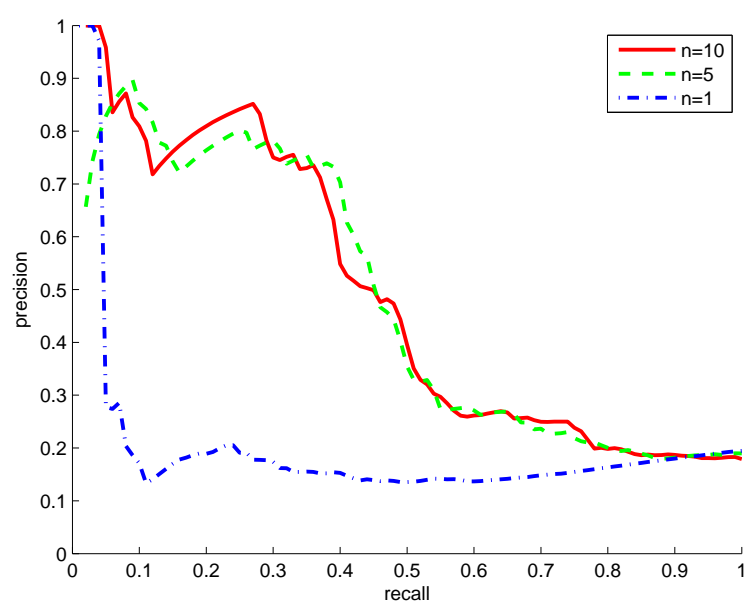

(b)

Figure 6: Performance for the saxophone class across different training set sizes. Graph (a) indicates results using our approach. Graph (b) indicates results without proper initialization methods. 


\subsection{The effect of training set size}

Training data represents costly human labor and naturally we wish to reduce it. In the web image search scenario, the training set is the set of images chosen by the user as desirable examples. This means that our model must succeed using only a few images as training data, or there will be too much work required by the user. Ideally the system would require only a single example for reasonable performance.

In figure 6 we compare the performance of the model under training set sizes $n=10, n=5$, and $\mathrm{n}=1$ for the saxophone class. We note that our approach still gives very reasonable performance with only one training example.

Figure 6(b) demonstrates the importance of proper initialization measures for the hybrid semisupervised learning procedure. In this case we do not train initial parameter estimates prior to the update procedure. Performance remains reasonable as long as the training set is sufficiently large. Under these circumstances the model no longer functions with just one training example.

\section{Conclusions}

We have presented a method for mining visual concepts from the web using minimal supervision. Our approach is built upon a novel probabilistic graphical model which combines image features and text features from associated html documents. We introduced a hybrid expectation maximization / expected gradient procedure for the model and showed that this semi-supervised approach gives better performance than a number of baseline tests. The model was applied to a dataset of musical instruments collected from the web, and the resulting rerankings vastly improved upon rankings given by Google image search. Top images from the reranked data have correct labelings and are suitable as datasets in computer vision research.

Also, we have contributed a new publicly available dataset to be used for research combining text and vision. The dataset contains 9 classes of musical instruments and 4487 documents in total. We provide the original text and our annotations for each document. Future research exploring web images and text will benefit from a known, shared set of data over which performance can be compared.

\section{Acknowledgments}

Chris Pal thanks Kodak Research and Microsoft Research for gifts that helped support this work.

\section{References}

[1] K. Barnard, P. Duygulu, N. de Freitas, D. Forsyth, D. Blei, and M. Jordan. Matching words and pictures. Journal of Machine Learning Research, 3:1107-1135, 2003.

[2] T. Berg and D. Forsyth. Animals on the Web. Computer Vision and Pattern Recognition, 2006 IEEE Computer Society Conference on, 2, 2006. 
[3] D. Blei, A. Ng, and M. Jordan. Latent Dirichlet allocation. Journal of Machine Learning Research, 3(5):993-1022, 2003.

[4] A. P. Dempster, N. M. Laird, and D. B. Rubin. Maximum likelihood from incomplete data via the EM algorithm. Journal of the Royal Statistical Society. Series B (Methodological), 39(1):1-38, 1977.

[5] G. Druck, C. Pal, J. Zhu, and A. McCallum. Semi-supervised classification with hybrid generative/discriminative methods. In Proceedings of Knowledge Discovery and Data Mining (KDD), 2007.

[6] R. Fergus, L. Fei-Fei, P. Perona, and A. Zisserman. Learning Object Categories from Googles Image Search. Computer Vision, 2005. ICCV 2005. Tenth IEEE International Conference on, $2,2005$.

[7] R. S. . S. T. R. . Z. Ghahramani. Optimization with em and expectation-conjugate-gradient. In International Conference on Machine Learning (ICML), 2003.

[8] J. Lasserre, C. M. Bishop, and T. Minka. Principled hybrids of generative and discriminative models. In In Proceedings 2006 IEEE Conference on Computer Vision and Pattern Recognition, New York, 2006.

[9] G. W. Li-Jia Li and L. Fei-Fei. Optimol: automatic object picture collection via incremental model learning. In IEEE Computer Vision and Pattern Recognition (CVPR), 2007.

[10] D. Lowe. Object recognition from local scale-invariant features. International Conference on Computer Vision, 2:1150-1157, 1999.

[11] A. McCallum, C. Pal, G. Druck, and X. Wang. Multi-conditional learning: Generative/discriminative training for clustering and classification. Proceedings of 21st National Conference on Artificial Intelligence (AAAI), 2006.

[12] A. K. McCallum. Mallet: A machine learning for language toolkit. http://mallet.cs.umass.edu, 2002 .

[13] A. Y. Ng and M. I. Jordan. On discriminative vs. generative classifiers: A comparison of logistic regression and naive bayes. In NIPS, pages 841-848, 2001.

[14] E. Nowak, F. Jurie, and B. Triggs. Sampling strategies for bag-of-features image classification. Proc. ECCV, 4:490-503, 2006.

[15] J. Ponce, T. Berg, M. Everingham, D. Forsyth, M. Hebert, S. Lazebnik, M. Marszalek, C. Schmid, B. Russell, A. Torralba, et al. Dataset Issues in Object Recognition. Toward Category-Level Object Recognition. LNCS, 4170.

[16] F. Schroff, A. Criminisi, and A. Zisserman. Harvesting image databases from the web. In Proceedings of the 11th International Conference on Computer Vision, Rio de Janeiro, Brazil, 2007. 
[17] Y. Teh, M. Jordan, M. Beal, and D. Blei. Hierarchical dirichlet processes. Journal of the American Statistical Association, 101(476):1566-1581, 2006.

[18] A. Torralba, R. Fergus, and W. T. Freeman. Tiny images. Technical Report MIT-CSAILTR-2007-024, Computer Science and Artificial Intelligence Lab, Massachusetts Institute of Technology, 2007. 